\title{
Painless labor with patient-controlled epidural analgesia protects against short-term pelvic floor dysfunction: a retrospective cohort study
}

\author{
Lijun Ruan ${ }^{1,2}$, Xiaowu $\mathrm{Xu}^{2}$, Hongen $\mathrm{Wu}^{2}$, Yue Xiao ${ }^{2}$, Wanling $\mathrm{Li}^{2}$, Haiyan Lin ${ }^{2}$, Limei Zheng ${ }^{2}$, Ying $\mathrm{Cai}^{3}$ \\ ${ }^{1}$ Guangzhou University of Traditional Chinese Medicine, Guangzhou, China; ${ }^{2}$ Department of Obstetrics and Gynecology, The Fifth Affiliated \\ Hospital of Southern Medical University, Guangzhou, China; ${ }^{3}$ Southern Medical University of China, Guangzhou, China \\ Contributions: (I) Conception and design: L Ruan; (II) Administrative support: X Xu; (III) Provision of study materials or patients: $\mathrm{H}$ Wu, W Li, H \\ Lin; (IV) Collection and assembly of data: L Ruan, Y Cai; (V) Data analysis and interpretation: L Zheng; (VI) Manuscript writing: All authors; (VII) \\ Final approval of manuscript: All authors. \\ Correspondence to: Xiaowu Xu. Chief Physician, Department of Obstetrics and Gynecology, The Fifth Affiliated Hospital of Southern Medical \\ University, 566 Congcheng Avenue, Conghua District, Guangzhou, China. Email: 2100390201@qq.com.
}

\begin{abstract}
Background: The aim of the present study was to investigate whether painless labor with patientcontrolled epidural analgesia (PCEA) has a protective effect on pelvic floor function. And to observe if there was any difference in the effect of painless labor with PCEA versus vaginal delivery on postpartum shortterm pelvic floor function.

Methods: All women who delivered at the hospital's obstetric department during June 2016 to October 2018 were included in the study. They were divided according to delivery mode: painless labor with PCEA [group A (observation group), $\mathrm{n}=27$ ], and vaginal delivery [group B (control group), n=36]. Pelvic floor function was assessed at postpartum 7 weeks.

Results: Groups A and B showed no significant difference in the total score at postpartum 6-8 weeks. However, group A showed lower pelvic floor muscle tone at rest and significantly higher muscle strength scores than group B. Both the pre-rest and post-rest phase muscle strength was stronger than in group B ( $\mathrm{P}=0.039$ and $\mathrm{P}=0.016$, respectively). There was no significant difference in pelvic floor muscle strength between analgesia and non-analgesia groups, or between episiotomy and non-episiotomy recipients.
\end{abstract}

Conclusions: Painless labor with PCEA reduced both pain during the delivery and injury to the pelvic floor. It had protective effect on the pelvic floor muscles.

Keywords: Painless labor with patient-controlled epidural analgesia; pelvic floor function; rest pelvic floor muscle tone; vaginal delivery

Submitted Jun 30, 2020. Accepted for publication Aug 25, 2020.

doi: 10.21037/apm-20-1430

View this article at: http://dx.doi.org/10.21037/apm-20-1430

\section{Introduction}

Pelvic floor dysfunction (PFD) refers to conditions caused by structural defects, injuries and dysfunction of the pelvic floor support, which may lead to stress urinary incontinence, overactive bladder, pelvic organ prolapse, and fecal incontinence (1). These conditions are common in women after childbirth and have a significant effect on quality of life, mental health, and sex life. The main treatment for PFD is rehabilitating the pelvic floor and modifying the tone of the pelvic floor muscles (PFMs).

The pathophysiology of PFD is complex, with an etiology that includes ligament, fascia, and muscle tears or injury during labor, early postpartum physical labor (especially heavy physical labor), chronic intra-abdominal hypertension, and iatrogenic reasons. Among these, pregnancy and delivery are the most important. Both 
maternal body weight and uterine weight increase during pregnancy, with stretching and overloading of the pelvic fascia, the cardinal ligament of the uterus, the utero-sacral ligament, and pelvic floor muscles. In addition, the enlarged uterus increases the lateral angle of the urethra, leading to bladder neck hypermobility and urinary tract symptoms (2). Epidemiologic research shows that pelvic floor function is associated with delivery and parity and dysfunction can occur after vaginal delivery or cesarean delivery (3). Urbankova et al. found that age, pre-pregnant body mass index, and body weight gain during pregnancy increased the risk of PFD (4), but vaginal delivery is the most common risk factor. Vaginal delivery directly affects the pelvic floor structure and tissues (5), especially during the second stage of delivery (6). Painless labor with patient-controlled epidural analgesia (PCEA) uses an epidural catheter that is connected to a pump that the participant can control by pressing a button to adjust the dosing of analgesic. Thus, a continuous small dose of analgesic can be administered during the whole labor, which helps to relax the pelvic floor tissues and prevents pelvic floor muscle injury due to the fear of pain, especially in primiparas. In addition, the participant can still walk around with an assistant.

The purpose of the present study was to investigate the effect of delivery mode on pelvic floor function at postpartum 6-8 weeks (average time for damaged pelvic muscle recovery) in primiparas. We compared pelvic floor function and analyzed the effect of painless labor on pelvic floor function in primiparas with vaginal delivery.

We present the following article in accordance with the STROBE reporting checklist (available at http://dx.doi. org/10.21037/apm-20-1430).

\section{Methods}

\section{Clinical data}

This was a single-center, retrospective cohort study including 506 primiparas who had a vaginal delivery between June 2016 and October 2018 at the Fifth Affiliation Hospital of the Southern Medical University and attended the hospital's pelvic floor rehabilitation center at postpartum 6-8 weeks. The inclusion criteria were: (I) primiparity, singleton, head presentation, and vaginal delivery; (II) no lochia; (III) no previous surgical history of the urogenital system; (IV) informed consent for pelvic floor function test; (V) no instruments used for labor assistance; and (VI) the participant had not performed any rehabilitation exercise.
Participants with the following conditions were excluded: (I) giant fetus; (II) diabetes; (III) neuromuscular diseases; and (IV) chronic cough, constipation, pelvic organ prolapse, or history of urinary incontinence. Delivery methods were chosen based on discussion of parturient with doctor. The study was conducted in accordance with the Declaration of Helsinki (as revised in 2013). Our study was approved by the institutional ethics committee (NYWY201601). All participants gave informed consent.

\section{Analgesia protocol}

PCEA used the same puncture site as for epidural anesthesia during cesarean section (i.e., L2-3 or L3-4). A mixture of ropivacaine and sufentanil was administered at $4 \mathrm{~mL} / \mathrm{h}$.

\section{Pelvic floor function test}

Detailed information of delivery mode and pelvic floor function was collected at postpartum 6 weeks (average time for damaged muscle recovery). A pelvic floor muscle electromyogram was recorded using a biofeedback electrostimulant therapy instrument (Medlander, Nanjing, China). The strength of the class I muscle fibers was recorded during a contraction of $10 \mathrm{~s}$. The strength of the class II muscle fibers was the mean value of five fast contractions. The resting pelvic floor muscle strength and the dynamic strength of the class II and class I muscles were evaluated.

\section{Statistical analysis}

All statistical analyses were performed using SPSS 20.0. All data were input simultaneously by two investigators. Continuous data are presented as mean and standard deviation. Categorical data are presented as percentages. Equality of variance of the continuous data was examined using the Levene's test. Data with equal variance were compared using the two-sample $t$-test, while those with unequal variance were compared using the rank-sum test. $\mathrm{P}<0.05$ or the false discovery rate $<0.05$ was considered statistically significant.

\section{Results}

\section{Baseline characteristics}

At postpartum 6 weeks, 27 participants with painless labor and 36 without any analgesia were evaluated. There was no 
Table 1 General information of the primiparas with and without analgesia (mean \pm standard deviation, $\mathrm{n}$ )

\begin{tabular}{|c|c|c|c|c|}
\hline Characteristics & Analgesia group $(n=27)$ & Non-analgesia group $(n=36)$ & $\mathrm{t}$ value & $P$ value \\
\hline Gestational week & $39.67 \pm 1.31$ & $39.14 \pm 1.10$ & 1.857 & 0.068 \\
\hline Body mass index & $21.39 \pm 2.20$ & $21.31 \pm 2.12$ & 0.155 & 0.878 \\
\hline Neonatal weight & $3.11 \pm 0.30$ & $3.06 \pm 0.30$ & 0.627 & 0.533 \\
\hline
\end{tabular}

Table 2 Pelvic floor muscle scores of the primiparas with and without analgesia (mean \pm standard deviation, $\mathrm{n}$ )

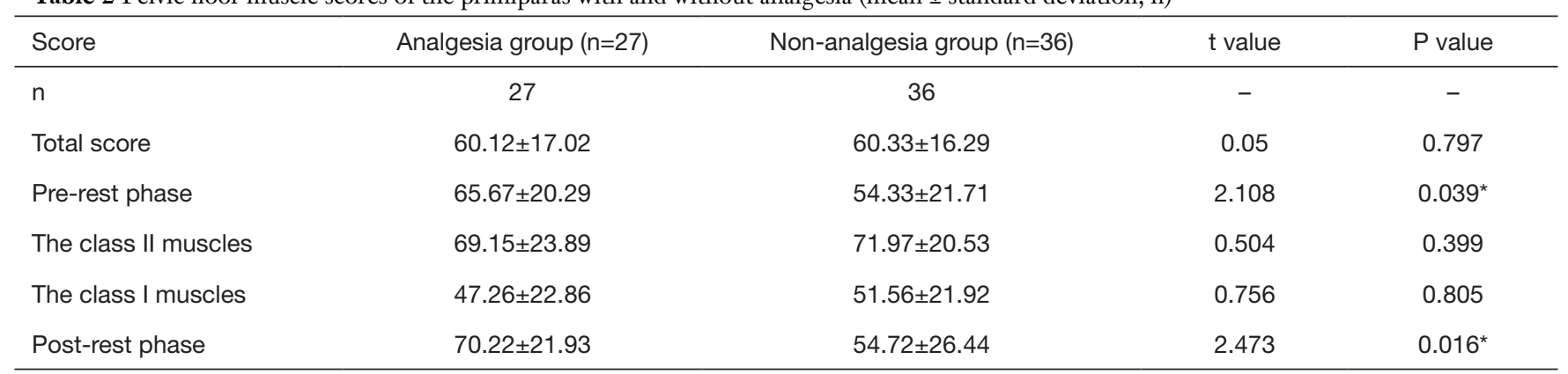

*, the scores in the pre- and post-rest phases at postpartum 6-8 weeks in primiparas with PCEA are higher than in those without analgesia. The difference was statistically significant.

Table 3 Distribution of lateral episiotomy and vaginal laceration in the analgesia group

\begin{tabular}{|c|c|c|c|c|}
\hline Group & Lateral episiotomy & Vaginal laceration & $\chi^{2}$ & $P$ value \\
\hline
\end{tabular}

significant difference between groups in the participants' general information $(\mathrm{P}>0.005$, Table 1$)$.

\section{Damage of the pelvic floor}

Both normal labor and painless labor damaged the pelvic floor, but participants showed varied levels of pelvic floor muscle fatigue, with no significant difference in the total score. Primiparas with painless labor had significantly better scores in the pre- and post-rest phases at postpartum 6-8 weeks than those without analgesia, but there was no significant difference in the class II or class I muscles between the two groups (Table 2). Two participants with a lateral episiotomy complicated by vaginal laceration were excluded, so there was no statistical difference $(\mathrm{P}=0.973)$ in the incidence of vulvar laceration and lateral episiotomy in the painless delivery group (Table 3). There was no significant difference in postpartum pelvic floor function between patients with perineal laceration and those with perineal incision in the painless labor group (Table 4).

\section{Discussion}

Our findings of higher scores in the pre- and post-rest phases at postpartum 6-8 weeks in primiparas with PCEA than in those without analgesia, as well as the lack of a significant difference between the two groups in the class II or the class I muscles, suggest that painless labor protected the pelvic floor muscles. Urbankova et al. (4) also found that epidural anesthesia protects pelvic floor function. The reference myoelectricity value is $2-4 \mu \mathrm{V}$ in the pre-rest phase when the muscles rest. In the post-rest phase, the pelvic floor muscles are relaxed. In the present study, the women without analgesia had significantly higher pre- and post-rest potentials than those with analgesia, suggesting there might be pelvic floor muscle overactivity, which requires low-frequency electrotherapy for muscle relaxation.

In the Department of Obstetrics of the Southern 
Table 4 Comparison of pelvic floor muscle scores between the primiparas with lateral episiotomy and those with vaginal lacerations

\begin{tabular}{|c|c|c|c|c|}
\hline Score & Lateral episiotomy $(n=14)$ & Vaginal laceration $(n=11)$ & $\mathrm{t}$ value & $P$ value \\
\hline Pre-rest phase & $67.50 \pm 19.93$ & $59.81 \pm 20.87$ & 0.93 & 0.35 \\
\hline Fast-twitch muscles (class II) & $66.85 \pm 27.45$ & $68.63 \pm 20.38$ & -0.17 & 0.85 \\
\hline Slow-twitch muscles (class I) & $44.00 \pm 28.73$ & $49.90 \pm 15.68$ & -0.61 & 0.54 \\
\hline
\end{tabular}

V3 with unequal variances were compared using the non-parametric test. $Z=-0.329, P=0.742$.

Medical University Fifth Hospital, the delivery volume is high and increasing every year. Since March 2014, we have been performing painless labor as a routine medical service. Anesthetists remain in the department to ensure patient safety, so we have gained a lot of experience with painless labor. Painless labor and dedicated labor wards improve patient comfort and the delivery, reducing the risks of fatigue, bleeding, difficult labor, cesarean section, and neonatal apnea. There are also benefits in postpartum recovery and bonding between the mother, baby, and family. Women with painless labor have a lower incidence of postpartum depression and better perinatal safety of both mother and baby. The benefits of painless labor include reduced hospital stay, medical costs, and familial and social economic burden. The cesarean rate in 2018 in China was $36.7 \%$, and among the many reasons for this high rate, fear of labor pain is an important one. Painless labor significantly reduces labor pain, cesarean rate, labor complications, and maternal mortality.

There are three main mechanisms of labor pain. First, the major reason for labor pain is the uterine contractions stretching or tearing the muscle fibers. Second, the fetus compresses the birth canal, especially the lower part of the uterus and the cervix, also causing stretching and pain. Third, fear and anxiety exaggerates the labor pain. Lack of labor experience in primiparas and misinformation about pain cause fear and muscular tension, leading to stronger and longer uterine contractions, and thus more labor pain. Although pain is the fifth vital sign, labor pain ranks first in pain scoring. It can result in maternal respiratory alkalosis, left shifting of the oxygen dissociation curve, spasm of uterine arteries, fetal hypoxia, and increased fetal oxygen consumption. In addition, labor pain affects the maternal psychology, leading to increased release of catecholamines and decreased lactation in primiparas (7). It has also been reported that labor pain is associated with early postpartum depression, which negatively affects maternal mental health and neonatal cognitive development $(8,9)$. Some women may choose cesarean delivery through fear of labor pain and the subsequent injuries. Painless labor is the goal of modern obstetric wards because it reduces maternal fear and the pain-associated complications.

The pelvic floor muscle is skeletal muscle, and according to the morphological and metabolic characteristics of muscle, it is divided into class I and class II fibers. Class I muscle fibers have a long contraction duration and do not easily fatigue. The deep pelvic floor muscle, which is mainly composed of this type of fiber, has a supporting function. The class II muscle fibers are fast in contraction and easily fatigue. The superficial pelvic floor muscles are mainly composed of this type of fiber, and play a role in controlling urine, defecation and sexual function. The postpartum pelvic floor function did not differ significantly between women with a lateral episiotomy and those with vaginal injuries. Pelvic organ prolapse (POP) refers to conditions that the pelvic organs prolapsed inside or outside the vagina.t has been suggested that lateral episiotomy can prevent $\mathrm{PFD}$, whereas spontaneous perineal laceration is a risk factor of POP (10). However, our study did not confirm these findings. We found no significant difference in the incidence of lateral incision and perineal laceration in the analgesia group, and there was no significant difference in the postpartum pelvic floor function score between the two groups.

Women should be informed about the risk of PFD involved in pregnancy and delivery, but cesarean section should not be chosen because of fear of this risk. The World Health Organization states that cesarean section can result in severe, sometime permanent, complications, disability or death, and is only indicated with concrete medical reasons (11). The pregnant woman should be provided with scientific evidence in the first prenatal consultation about 
the delivery mode. The mother should be asked about PFD during the postpartum clinical visit and at postpartum 6 weeks by both the practitioners and midwifes, and preventive solutions be recommended if necessary.

Limitations of our study are the small sample size and the relatively inadequate data. Prospectively and constantly monitoring the changes in pelvic floor function in primiparas with painless labor may help to better understand the protective effect of painless labor on the pelvic floor muscles. We are planning to continue follow-up of our participants and collect data on pelvic floor function and quality of life.

\section{Conclusions}

Changes in pelvic floor electrophysiology are closely related to the occurrence and development of PFD. Moreover, these changes often occur before the clinical symptoms of PFD. Therefore, electrophysiological examination of the pelvic floor can detect injury in the early stage, enabling individual analysis and judgment according to the results of the examination, and a subsequent individual rehabilitation treatment plan. In this study, we found that tension of pelvic floor muscles decreased and the symptoms of pelvic floor ischemia were alleviated in the painless delivery group, which protected the function of pelvic floor muscles to a certain extent. Early postpartum (6 weeks to 3 months postpartum) is the most vulnerable period for the woman, and also the best period for rehabilitation. It is hoped that even under basic measures of pelvic floor rehabilitation, different programs will be selected according to different delivery methods.

\section{Acknowledgments}

Funding: This project was funded by the Scientific Research Project of Guangdong Administration of Traditional Chinese Medicine (20172100); Scientific Research Project of Guangdong Administration of Traditional Chinese Medicine (20181180); Scientific Research Project of Chinese Preventive Medicine Association (201819123).

\section{Footnote}

Reporting Checklist: The authors have completed the STROBE reporting checklist. Available at http://dx.doi. org/10.21037/apm-20-1430

Data Sharing Statement: Available at http://dx.doi. org/10.21037/apm-20-1430

Conflicts of Interest: All authors have completed the ICMJE uniform disclosure form (available at http://dx.doi. org/10.21037/apm-20-1430). The authors have no conflicts of interest to declare.

Ethical Statement: The authors are accountable for all aspects of the work in ensuring that questions related to the accuracy or integrity of any part of the work are appropriately investigated and resolved. The study was conducted in accordance with the Declaration of Helsinki (as revised in 2013). Ethical approval of the study was obtained from the Ethics Committee of The Fifth Affiliated Hospital of Southern Medical University (NYWY201601) on 07/07/2016. All participants gave informed consent.

Open Access Statement: This is an Open Access article distributed in accordance with the Creative Commons Attribution-NonCommercial-NoDerivs 4.0 International License (CC BY-NC-ND 4.0), which permits the noncommercial replication and distribution of the article with the strict proviso that no changes or edits are made and the original work is properly cited (including links to both the formal publication through the relevant DOI and the license). See: https://creativecommons.org/licenses/by-nc-nd/4.0/.

\section{References}

1. Dietz HP. Pelvic floor trauma in childbirth. Aust N Z J Obstet Gynaecol 2013;53:220-30.

2. Herbert J. Pregnancy and childbirth: the effects on pelvic floor muscles. Nurs Times 2009;105:38-41.

3. Handa VL, Blomquist JL, Knoepp LR, et al. Pelvic floor disorders 5-10 years after vaginal or cesarean childbirth. Obstet Gynecol 2011;118:777-84.

4. Urbankova I, Grohregin K, Hanacek J, et al. The effect of the first vaginal birth on pelvic floor anatomy and dysfunction. Int Urogynecol J 2019;30:1689-96.

5. Callewaert G, Albersen M, Janssen K, et al. The impact of vaginal delivery on pelvic floor function - delivery as a time point for secondary prevention. BJOG 2016;123:678-81. 
6. Memon HU, Blomquist JL, Dietz HP, et al. Comparison of levator ani muscle avulsion injury after forceps-assisted and vacuum-assisted vaginal childbirth. Obstet Gynecol 2015;125:1080-7.

7. Al-Tamimi Y, Ilett KF, Paech MJ, et al. Estimation of infant dose and exposure to pethidine and norpethidine via breast milk following patient-controlled epidural pethidine for analgesia post caesarean delivery. Int J Obstet Anesth. 2011;20:128-34.

8. Eisenach JC, Pan PH, Smiley R, et al. Severity of acute pain after childbirth, but not type of delivery, predicts persistent pain and postpartum depression. Pain.

Cite this article as: Ruan L, Xu X, Wu H, Xiao Y, Li W, Lin H, Zheng L, Cai Y. Painless labor with patient-controlled epidural analgesia protects against short-term pelvic floor dysfunction: a retrospective cohort study. Ann Palliat Med 2020;9(5):3326-3331. doi: 10.21037/apm-20-1430
2008;140:87-94.

9. Field T. Postpartum depression effects on early interactions, parenting, and safety practices: a review. Infant Behav Dev 2010;33:1-6.

10. Memon H, Handa VL. Pelvic floor disorders following vaginal or cesarean delivery. Curr Opin Obstet Gynecol 2012;24:349-54.

11. Betran AP, Torloni MR, Zhang JJ, et al. WHO Statement on Caesarean Section Rates. BJOG 2016;123:667-70.

(English Language Editor: K. Brown) 